Archives

$10 \mid 1993$

Le Centre de recherches historiques de 1949 à 1975

\title{
Le profil d'une institution
}

\section{OpenEdition}

Journals

Édition électronique

URL : http://journals.openedition.org/ccrh/2787

DOI : $10.4000 /$ ccrh. 2787

ISSN : $1760-7906$

Éditeur

Centre de recherches historiques - EHESS

Édition imprimée

Date de publication : 15 avril 1993

ISSN : 0990-9141

Référence électronique

"Le profil d'une institution », Les Cahiers du Centre de Recherches Historiques [En ligne], 10 | 1993, mis en ligne le 16 mars 2009, consulté le 20 avril 2019. URL : http://journals.openedition.org/ccrh/2787 ; DOI : $10.4000 /$ ccrh. 2787

Ce document a été généré automatiquement le 20 avril 2019.

Article L.111-1 du Code de la propriété intellectuelle. 


\section{Le profil d'une institution}

1 Avant d'analyser en détail le développement des activités de recherche du CRH, il me semble utile de résumer les grandes lignes de l'évolution institutionnelle du laboratoire. Les structures d'organisation qui se sont cristallisées pendant la phase longue d'expansion n'ont cessé de s'alourdir ni de peser sur les stratégies de recherche de ses membres. Au moins trois facteurs ont façonné le profil institutionnel du CRH : sa place dans l'organigramme de la $\mathrm{VI}^{\mathrm{e}}$ Section, dans la division du travail scientifique et du pouvoir en son sein; le système de recrutement de son personnel de recherche; et l'évolution de l'enseignement dans la division d'histoire de la VI Section qui lui est restée étroitement liée.

Pendant les années 1950, le CRH était intégré dans un organigramme ternaire simple: histoire - économie - sociologie/anthropologie. En face d'études économiques et sociologiques plus dispersées et plus liées à des centres extérieurs à la VI ${ }^{e}$ Section, le CRH restait la première et seule institution de la Section qui avait l'ambition d'unifier ou de coordonner toutes les recherches dans «sa » division. Cette différence entre les secteurs de la $\mathrm{VI}^{\mathrm{e}}$ Section, qui s'explique par les tensions à l'intérieur du champ de recherches économiques et par les ambiguités du statut de la sociologie française des années $1950^{1}$, a survécu aux hasards événementiels de l'époque. Aujourd'hui encore, le CRH est de loin le plus grand parmi la myriade de centres spécialisés qui caractérise actuellement l'EHESS, et, par conséquent, il cherche à fédérer un champ de recherches très dispersées à l'intérieur du monde historiographique de l'École - une ambition qui est largement inconnue des autres centres de l'école.

3 L'élargissement du champ des recherches et des enseignements de la VI ${ }^{\mathrm{e}}$ Section a tout de suite brouillé l'organigramme ternaire initial. Avec la mise en route du programme des «aires culturelles» en 1955-56. L'apparition d'une histoire extra-européenne de plein droit, qui fut sans aucun doute l'une des innovations les plus marquantes de la $\mathrm{VI}^{\mathrm{e}}$ Section, limitait l'expansion géographique du CRH. Par conséquent, les historiens de la Chine, de l'Afrique noire ou de l'Inde recrutés à partir de 1955, comme Balacz, Chesneaux, Gernet ou Elisseeff, plus tard Portal, Besançon, Haupt, Thorner, Bennigsen, Brunschwig ou Holzman, sont restés hors de l'orbite du CRH. La frontière entre les domaines de recherche des aires culturelles et du Centre de recherche historiques n'a jamais été fixée d'une manière définitive et elle n'a jamais été une cloison étanche, mais elle a tenu à 
distance du CRH toute une série de terrains spéciaux de la recherche historique, pourtant bien proches des siens, comme par exemple les recherches du Centre d'Etudes juives, l'histoire de la Russie et du monde islamique ou l'histoire du Marxisme. La création de la division des "aires culturelles» a favorisé l'indépendance institutionnelle de tous ces groupes définis par des domaines bien délimités. Cette expansion thématique et géographique de la $\mathrm{VI}^{e}$ Section accompagnée d'une croissance de ses ressources financières incita d'autres groupes à chercher leur indépendance institutionnelle. Ainsi, en 1958 le centre de recherche d'histoire des sciences et des techniques était fondé par A. Koyré, puis en 1959 le Centre d'Études pré- et protohistoriques par Varagnac, centres qui, pour des raisons budgétaires, restaient affiliés au CRH mais qui gardaient leur autonomie institutionnelle et leur indépendance intellectuelle.

Le seul continent extra-européen conquis par le CRH est l'Amérique Latine. Cette annexion d'ailleurs hésitante et finalement arrêtée à mi-chemin était inscrite dans la logique des recherches inspirées par Braudel et par sa thèse sur la Méditerranée. Mais dans ce cas, la concurrence d'autres centres comme celui de l'Institut des Hautes Études de l'Amérique Latine de l'université de Paris ou de l'Institut d'Études Hispaniques, Hispano-américaines et Luso-Brésiliennes de Toulouse, a sans doute freiné l'expansionnisme du Centre. Les orientations du premier directeur ont marqué à long terme d'autres frontières du centre: le monde byzantin faisant partie du patrimoine méditerranéen, l'archéologie surtout médiévale ouvrant des voies nouvelles pour la découverte de la civilisation matérielle - ces champs de recherche sont restés annexés plus durablement après les partages internes de 1955-56.

5 L'histoire ancienne présente un cas spécifique dans l'organigramme de la $\mathrm{VI}^{\mathrm{e}}$ Section. Ses premiers directeurs d'études étaient dispersés entre l'histoire (J. Guey) et la sociologie (J. P. Vernant, A. Aymard) et ce n'est qu'à partir de 1964 que s'est formé le groupe de J. P. Vernant et $P$. Vidal-Naquet qui animaient une série de séminaires au CRH avant de fonder un peu plus tard le Centre de Recherches Comparées sur les Sociétés anciennes. Marqué par un héritage intellectuel spécifique (Gernet et Meyerson), ce groupe n'était qu'un hôte de passage du CRH et gagna son autonomie institutionnelle au début des années $1970^{2}$.

6 La tendance typique pour la $\mathrm{VI}^{\mathrm{e}}$ Section consistait à gérer son expansion par une structure "sérielle " souple - ajoutant aux foyers de recherche existants de nouveaux centres, ce qui fait naître à chaque élection d'un nouveau directeur d'études un centre de recherche virtuel - était comme en suspens à l'intérieur de la division d'histoire tout au long des années 1960. Le patriotisme d'institution et une politique énergique de centralisation a permis au CRH d'intégrer la plupart des historiens nouvellement recrutés et de maintenir l'unité organisationnelle d'un personnel qui commençait à s'engager dans des champs de recherche toujours plus distants. Mais la dernière étape d'expansion à partir de 1969 a renforcé les tendances centrifuges sous-jacentes et en 1974-75, la division d'histoire de l'EHESS comptait 10 centres de recherche qui survivent à la mauvaise conjoncture scientifique des années suivantes et qui sont toujours présents en 1984 - sauf le Laboratoire de Démographie historique réintégré au CRH en 1979. Qu'il s'agisse du Centre de Documentation d'Histoire des Techniques, du Centre de Sociologie des Objets de Civilisation, du Centre de Recherches comparées sur les Sociétés anciennes ou du Groupe de Recherche Cinéma et Histoire ou du Centre d'Anthropologie religieuse Européenne, tous ces petits centres ont préféré leur autonomie institutionnelle et leur visibilité symbolique à l'intégration dans un organisme de recherche qui semble avoir 
atteint les limites de sa croissance si l'on en juge par ses capacités d'intégration conceptuelle.

Tableau 3. Évolution du personnel scientifique du CRH

\begin{tabular}{|c|c|c|c|c|c|c|c|c|}
\hline Fonctions & 1952 & 1957 & 1962 & 1965 & 1968 & 1971 & 1974 & 1979 \\
\hline $\begin{array}{l}\text { Dir. d'Etud. } \\
\text { M.de Rech. }\end{array}$ & $2(0)$ & $4(0)$ & $5(0)$ & $7(0)$ & $9(0)$ & $18(0)$ & $18(0)$ & $\begin{array}{c}23+2 \\
(1)\end{array}$ \\
\hline Ss .Dir. d'Et. & $\because$ & $=$ & 201 & $2(0)$ & $3(0)$ & $4(0)$ & $5(0)$ & 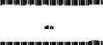 \\
\hline M-Assit. & $\because$ & $=$ & $10)$ & $3(0)$ & $5(0)$ & $6(2)$ & $14(4)$ & 217 \\
\hline $\begin{array}{l}\text { Chefs de } \\
\text { Travaux }\end{array}$ & - & $2(0)$ & $16(6)$ & $19(9)$ & $\begin{array}{l}23 \\
\text { (11) }\end{array}$ & $\begin{array}{c}22 \\
(11)\end{array}$ & $\begin{array}{l}23 \\
9\end{array}$ & $\begin{array}{l}11 \\
\text { (4) }\end{array}$ \\
\hline Ch, de Rech. & - & $=$ & $\because$ & 20 & 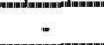 & $=$ & $\because$ & $4(1)$ \\
\hline Att.de Rech. & $13(1)$ & - & $\because$ & $=$ & - & - & $\because$ & $9(2)$ \\
\hline Ingén.CNRS & - & 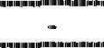 & 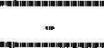 & $\cdots$ & $2(2)$ & $3(3)$ & $3(3)$ & 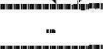 \\
\hline $\begin{array}{l}\text { Collaborat. } \\
\text { techn. ONRS }\end{array}$ & $6(4)$ & $6(6)$ & $5(2)$ & $4(4)$ & $10(1)$ & $9(6)$ & $6(5)$ & $\begin{array}{c}19 \\
(16)\end{array}$ \\
\hline $\begin{array}{l}\text { Agents } \\
\text { contractuels }\end{array}$ & 2 & $=$ & $\because$ & $1(0)$ & 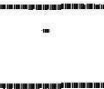 & $5(4)$ & $5(4)$ & $\begin{array}{c}18 \\
(14)\end{array}$ \\
\hline Préparateurs & $=$ & $=$ & $4(3)$ & $3(2)$ & 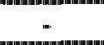 & $3(3)$ & $3(3)$ & 1 \\
\hline Vacataires & - & 24 & 14 & 35 & $\begin{array}{c}27 \\
(20)\end{array}$ & $\begin{array}{c}19 \\
(14)\end{array}$ & $\begin{array}{c}20 \\
(14)\end{array}$ & $\begin{array}{l}6 \\
(6)\end{array}$ \\
\hline Total & 23 & 36 & 47 & 76 & 79 & 89 & 97 & 114 \\
\hline
\end{tabular}

Les chiffres entre parenthèses indiquent le nombre de femmes. Sources : Archives EHESS Fonds Velay : rapports d'activités du CRH 1952-53, 1968, 1971, 1972-74, 1978-80; EPHE VI Section, budgets 1953 à 1975 ; rapports avec le CNRS 102 No 68. Les indications des rapports d'activités ont été vérifiées par une comparaison avec les chiffres des budgets annuels de l'Ecole mais il reste toujours des erreurs qu'on n'aurait pu éviter qu'en ayant recours aux dossiers des personnels de l'EHESS ou du CNRS.

7 En comparant les structures du CRH entre 1949 et 1975 il faut toujours tenir compte du fait que ce centre nominalement identique a subi des mutations profondes par les seuls effets de sa croissance. Le «laboratoire de recherches historiques » animé par un petit cercle de chercheurs autour de F. Braudel dont parlent les premiers rapports d'activité, a cédé la place à une institution qui intègre une centaine de chercheurs qui forment des groupes et des réseaux informels à travers les différentes strates de la hiérarchie officielle. À la lumière des seuls chiffres du tableau 3 un discours abstrait sur les tendances à l'émiettement et la perte d'identité du Centre qui oublie en même temps de parler des effets morphologiques de la croissance relève de la pure polémique: les conditions de communication à l'intérieur du Centre, les techniques de direction et de coordination scientifiques, toute la vie intérieure du CRH ont forcément changé au cours de cette expansion vertigineuse.

Les chiffres de ce tableau permettent d'évaluer le poids de l'institution et de mesurer sa croissance, qui reste un fait structurel constant jusqu'au début des années 1970, malgré son changement de rythme au cours des 25 dernières années. Il faut attendre la fin des années 1950 pour voir commencer la période d'expansion rapide du personnel avec la création de 16 postes de chef de travaux en 1960 et plus lentement l'augmentation des postes de directeurs d'études. Le CRH a besoin de 15 ans pour passer de ses 21 chercheurs de 1952 aux 52 mentionnés dans le premier rapport d'activité du laboratoire associé no 93 du CNRS de 1966. Mais c'est essentiellement le «boom» entre 1967 et 1972 qui change profondément l'échelle de grandeur. C'est en haut de la hiérarchie interne que les effets de l'expansion sont les plus notables: les directeurs d'études liés au CRH passent de 9 à 
18, ce doublement du nombre des chercheurs enseignants hiérarchiquement indépendants imposant de considérer que l'institution du début des années 1970 n'a plus grand chose à voir avec l'institution des années 1950, dirigée par un maître incontesté assisté par un personnel statutairement ou intellectuellement dépendant de lui.

9 Si l'on cherche à interpréter ces chiffres, qui ne sont d'ailleurs pas toujours fiables (surtout celui des vacataires), on peut déceler au moins une constante. Tout au long de ses 25 premières années, le CRH restait marqué par une stricte division hiérarchique des postes selon le sexe : les postes largement féminisés se trouvaient au bas de la hiérarchie. Les femmes étaient majoritaires au CRH parmi les vacataires, les préparateurs et les collaborateurs techniques, elles occupaient les postes qui sont statutairement des postes d'exécution, de spécialisation technique ou d'assistance. Le domaine de l'enseignement, de la direction ou de la synthèse de recherche et celui de l'écriture ou de la présentation de la recherche est resté l'apanage des hommes.

10 La ligne de partage entre les deux terrains traversait la catégorie des chefs de travaux : on peut y observer l'arrivée d'un nombre croissant de femmes pendant la période d'expansion des années 1960 qui conduisit à une répartition égale entre les deux sexes en 1971. Cette féminisation ne se poursuit plus après la fin de la conjoncture d'expansion des postes: en 1979, l'attribution des postes en fonction du sexe reste la règle mais elle connaît beaucoup plus d'exceptions que 20 ans auparavant. La comparaison des groupes de chefs de travaux et de maîtres-assistants recrutés au CRH jusqu'en 1975 fait apparaître des différences de carrière très marquées selon le sexe : la durée moyenne au poste de chef de travaux varie de plus de 3 ans et demi entre les deux sexes, elle est de 8,8 ans pour les 20 chefs de travaux masculins et de 12,23 ans pour les 17 chefs de travaux féminins français pris en compte ${ }^{3}$, respectivement 4 et 6 d'entre eux et d'entre elles se trouvaient encore à ce niveau hiérarchique en fin de carrière ou en 1985. Même si elles étaient arrivées à un poste de maître-assistant, les femmes du CRH ne franchissaient pas la dernière étape : l'élection à une direction d'études. Ce n'est qu'à la fin de l'époque considérée qu'on voit arriver les premières directrices d'études au CRH : C. KlapischZuber en 1979, L. Valensi en 1980. Dans les groupes de chefs de travaux et de maitresassistants nommés entre 1958 et 1975, 8 hommes avaient obtenu leur bâton de maréchal à la VI Section en 1985. Ces différences dans les carrières des chefs de travaux apparaissent d'autant plus nettes si l'on tient compte du fait que la possession du titre traditionnel d'excellence dans le champ historique, celui d'agrégé-normalien, n'intervient que dans cinq cas ( 2 femmes et 3 hommes). En fait, la nomination à un poste de chef de travaux se situait bien différemment dans le cursus honorum des chercheurs du CRH selon qu'ils étaient hommes ou femmes. Pour certaines, c'était l'aboutissement d'une carrière commencée dans des postes subalternes (vacataires, assistantes de recherche, préparatrices) ou dans le sillage de celle de leurs maris entrés comme directeurs d'études ( 6 des 20 femmes ont 39 ans et plus au moment de leur nomination), alors que pour les hommes et pour les autres femmes, la nomination au poste de chef de travaux représentait une première étape dans une carrière de chercheur qui, à l'époque, était encore très mal définie mais qui impliquait la promesse de promotions jusqu'au poste ultime de directeur d'études.

11 Les chiffres du tableau 4 font apparaître un autre trait spécifique du personnel de recherche au CRH : il s'agit de plus en plus d'un personnel permanent de titulaires. C'est en 1960, avec la création massive des postes de chefs de travaux, que débute cette évolution qui influe profondément sur les structures internes du Centre. En effet, l'âge 
moyen du personnel et l'ancienneté dans l'institution tendent à s'élever au cours de la période, bien que la création continue de postes assure l'entrée régulière de nouveaux chercheurs. En même temps, la différence d'âge entre les catégories hiérarchiques continue de baisser. En 1975, elle est d'un peu moins de 7 ans entre le groupe des directeurs et sous-directeurs d'études et celui des chefs de travaux et maîtres-assistants, l'expérience professionnelle au sein du CRH est pratiquement la même entre les deux catégories. A partir de 1960, les activités du Centre reposent sur un corps de chercheurs permanents qui s'homogénéise en termes d'âge et de fidélité à l'institution malgré les barrières hiérarchiques et les différences de carrière.

Tableau 4. Âge moyen et ancienneté des chercheurs au CRH

\begin{tabular}{|c|c|c|c|c|c|c|}
\hline Age nnoyen. & 1960 & Ni & 1968 & N & 1975 & N \\
\hline A. Ch de trav. & 36,9 & 16 & 37,3 & 27 & 4.0.1 & 16 \\
\hline M.-Assist. & $=$ & $\therefore$ & 36,3 & 3 & 42,7 & 15 \\
\hline Ensemble & 36,9 & 16 & 37,2 & 30 & 41,4 & 31 \\
\hline $\begin{array}{l}\text { Bi. Dir. et Ss. Dir. } \\
\text { d'études }\end{array}$ & 47,4 & 5 & 47,5 & 10 & 47,9 & 14 \\
\hline $\begin{array}{c}\text { Différences d'âge } \\
B-A\end{array}$ & 10,5 & & 10,3 & & 6,5 & \\
\hline Anciennete & 1960 & N & 1968 & $\mathrm{~N}$ & 1975 & N \\
\hline A. Ch de trav. & 0,4 & 16 & 4,3 & 27 & 8 & 16 \\
\hline M.-Assist. & $\therefore$ & - & 4 & 3 & 10,8 & 15 \\
\hline Ensemble & 0,4 & 16 & 4.3 & 30 & 9.3 & 31 \\
\hline $\begin{array}{l}\text { A Dir. et Ss. Dir. } \\
\text { d'érudes }\end{array}$ & 6,4 & 5 & 7,1 & 10 & 10.8 & 14 \\
\hline $\begin{array}{l}\text { Différences } \\
\text { d'ancienneté } \mathrm{B}-\mathrm{A}\end{array}$ & 6 & & 2,8 & & 1.5 & \\
\hline
\end{tabular}

Seules les dates concernant les chefs de travaux, maîtres-assistants et directeurs d'études effectivement attachés au CRH ont été retenues. L'ancienneté est calculée sur toutes les années d'activité à un poste permanent (y compris la période précédant la titularisation) : faute d'indications suffisantes, ces chiffres ne tiennent pas compte des années de vacation.

Ces résultats s'expliquent comme l'effet conjugué de deux évolutions. D'abord, le recrutement d'un personnel de chercheurs à statut de fonctionnaire titulaire induit à partir de 1960 une tendance au vieillissement et à la stabilité. Cet effet était contrebalancé jusqu'au début des années 1970 par celui de l'expansion qui permettait le recrutement de nouveaux collaborateurs plus jeunes sur des postes nouvellement créés. Les conditions favorables de la croissance incitaient aussi à poursuivre simultanément une politique d'avancement interne et d'intégration externe. Des 22 chefs de travaux recrutés pour la division « histoire » entre 1958 et 1960, 13 appartenaient encore à la VI ${ }^{\mathrm{e}}$ Section en 1975, deux autres venaient de prendre leur retraite. Si l'on ajoute les six chefs de travaux toujours présents en 1975, sur les neuf recrutés entre 1961 et 1964, on voit bien la persistance des individus dans cette catégorie centrale de chercheurs. Dans le groupe des directeurs d'études, la tendance au vieillissement lent en phase d'expansion s'explique par l'effet des postes de cumulants. Presque tous les directeurs d'études ont conservé leur poste à la $\mathrm{VI}^{\mathrm{e}}$ Section après avoir été nommés à un autre poste universitaire. Chaque vague de recrutement a ainsi déposé une autre strate de collaborateurs qui s'ajoutaient à ceux laissés par les vagues précédentes. Seulement 4 des 29 directeurs et sous-directeurs d'études de la division d'histoire élus entre 1947 et 1975 n'exerçaient plus leurs fonctions en 1975 : M. Lombard et J. Meuvret, morts en 1965 et 1971, J. Bouvier et R. Philippe ayant 
cessé leurs fonctions en $1964^{4}$. Il en est résulté une coexistence, parfois conflictuelle, de chercheurs marqués par les différentes phases du CRH, avec leurs styles de travail et leurs habitudes intellectuelles propres, et pour qui leur collaboration au CRH n'a représenté qu'une étape dans leur carrière universitaire et leur itinéraire intellectuel. Tel est le cas de R. Romano, actif au CRH entre 1951 et 1965, de Glénisson, entre 1958 et 1965, ou de D. Ozanam, entre 1956 et 1963.

13 La coexistence des générations et les différences de définition des postes de directeur d'études cumulant et non cumulant expliquent, en grande partie, une autre constante de l'existence du CRH : n'avoir jamais pu fédérer tous les directeurs d'études de la division d'histoire. Hormis les rivalités et les conflits personnels provenant d'une collaboration antérieure au CRH (par exemple Romano et Mandrou), cette carence provenait essentiellement $d u$ fait que le centre d'activités des directeurs cumulants se situait souvent hors de la VI ${ }^{e}$ Section ou d'un style de travail plutôt individualiste qui empêchait toute participation à une recherche collective qui, thématiquement, leur était pourtant souvent très proche. Tel fut le cas, par exemple, de directeurs d'études comme Meuvret, Lombard, Vilar, Goubert, Labrousse ou Leuillot, qui collaborèrent, au moins indirectement par leurs séminaires, aux activités scientifiques du CRH.

Bien que son activité, institutionnellement définie, fût la recherche et qu'il ne figurât pas dans le programme d'enseignement de la $\mathrm{VI}^{\mathrm{e}}$ Section, le profil du CRH porta l'empreinte de la liaison étroite entre recherche et enseignement qui est demeuré l'un des traits originaux de l'École dans le système d'enseignement supérieur français.

En fait, le Centre de recherches historiques participait plus ou moins directement à l'évolution de l'enseignement historique à la $\mathrm{VI}^{\mathrm{e}}$ Section. D'abord, l'orientation thématique des recherches du CRH se retrouve dans les programmes d'enseignement pourtant toujours plus larges et plus diversifiés de la division d'histoire. L'identité partielle du personnel et l'engagement intellectuel de sa majorité à l'entreprise des Annales ESC a contribué à effacer les différences et les distances entre les deux secteurs - l'enseignement de la division d'histoire et la recherche du CRH -, amalgamant par un autre effet d'institution l'image publique des deux. Celle-ci a souvent fait oublier l'existence de cours très spécialisés reflétant l'activité individuelle de directeurs d'études qui attiraient une audience de spécialistes peu liés aux chercheurs du CRH ou des autres centres de la VI Section.

L'éventail de l'enseignement de la division d'histoire s'est considérablement élargi tout au long de l'expansion de la $\mathrm{VI}^{\mathrm{e}}$ Section. Derrière la façade des regroupements thématiques et des changements de titre, on peut relever quelques tendances générales. Les années 1950 furent marquées par le choix fondamental de promouvoir l'histoire économique encore marginale dans l'enseignement universitaire. Les cours de J. Meuvret, E. Labrousse, P.Vilar, R. Romano, F. Braudel et de M. Lombard traitaient de sujets d'histoire économique et sociale de la fin de l'antiquité jusqu'à la Révolution. À partir de 1958, J. Guey élargit cet éventail avec ses cours sur la numismatique et l'histoire économique de l'antiquité. Une histoire sociale ouverte aux phénomènes culturels fut promue par toute une série de nominations à partir de 1960, celles de R. Mandrou (Histoire sociale des mentalités modernes), d'A Aymard (Sociologie de l'antiquité), de J.P. Vernant (Pensée sociale et religieuse de la Grèce ancienne) en 1958 et d A. Dupront (Psychologie collective et histoire de la civilisation européenne) en 1960, suivies par celles de Le Goff (Histoire et sociologie de l'occident médiéval, 1962), Jean Delumeau (Histoire et sociologie de l'Occident moderne, 1963) et A. Tenenti (Histoire sociale des cultures 
européennes, 1965). Mais il est typique de cette phase de transition que les cours d'Aymard, Vernant et Dupront s'inscrivirent dans le programme d'enseignement de la division de sociologie jusqu'au milieu des années 1960. L'enseignement de P. Goubert, d'E. Le Roy Ladurie et de P. Jeannin restaient plus proche de l'orientation antérieure vers une histoire socio-économique de l'époque moderne. L'intégration des cours d'archéologie de P. Courbin (Archéologie historique et civilisation matérielle 1960/1965) et d'histoire byzantine d'A. Guillou à partir de 1970) dans la division d'histoire allaient de pair avec celle de ces terrains au domaine de recherche du CRH. Les nominations à fin des années 1960 et au début des années 1970 furent l'occasion de réduire le poids d'une histoire économique et sociale qui avait marqué les origines de l'institution. Les cours de D. Richet sur l'histoire sociale et politique du XvI ${ }^{\mathrm{e}}$ siècle, ceux de F. Furet sur la culture et la politique dans la France du XVIII ${ }^{e}$ siècle, de J. Ozouf sur les comportements sociopolitiques dans la France contemporaine témoignent de ce changement d'orientation. Le domaine du politique réapparaissait, E. Labrousse et P. Vilar consacrant une partie de leurs cours à l'histoire du mouvement ouvrier et à des problèmes d'histoire politique. Après départ à la retraite des directeurs d'études de la première heure, l'histoire économique et sociale était représentée par P. Jeannin et L. Bergeron (Capitalisme et société en Europe occidentale, 1970) qui lui faisaient passer la frontière temporelle du XVIII ${ }^{\mathrm{e}}$ siècle jusqu'alors plutôt hermétique.

17 La liaison thématique recouvre des relations concrètes bien hétérogènes entre recherche et enseignement à l'intérieur de la $\mathrm{VI}^{\mathrm{e}}$ Section. Certains cours donnés par des directeurs prestigieux orientaient méthodologiquement et thématiquement les enquêtes du CRH. Le poids de l'enseignement des maîtres de la nouvelle histoire économique de l'époque moderne - E. Labrousse, F. Braudel, J. Meuvret - se faisait sentir surtout au début, lorsque l'écart d'âge et de statut entre les chercheurs du CRH et les directeurs de la division d'histoire était plus grand et la participation directe des derniers aux enquêtes plus rare. Ultérieurement, les cours de P. Goubert et de P. Vilar semblent avoir fonctionné de la même manière pour des groupes différents de chercheurs et d'étudiants engagés dans les enquêtes du CRH. Au cours des années 1960, avec la mise en chantier d'enquêtes plus vastes, apparurent les premiers séminaires de recherche liés aux activités des équipes du CRH groupés autour d'un directeur d'études. Les cours de F. Furet ou de J. Le Goff furent les premiers exemples de liaison directe entre recherche et enseignement.

18 Un autre point de liaison entre recherche et enseignement était, et demeure, la participation des chercheurs du CRH aux cours de la division d'histoire. Lorsque les annuaires de la $\mathrm{VI}^{\mathrm{e}}$ Section donnent des renseignements sur l'auditoire des cours et aux activités, on retrouve toujours ce groupe qui a une position intermédiaire entre les directeurs d'études et les autres auditeurs et constitue un relais de communication entre eux. Ils forment le groupe le plus actif aux conférences, ils assurent souvent la continuité des séminaires pluriannuels.

19 Par l'enseignement de la division d'histoire, les recherches du CRH trouvaient un premier écho et une première diffusion dans le monde universitaire. Bien qu'une statistique complète des étudiants et des auditeurs manque, les informations sur l'audience des cours donnent l'impression que celle-ci resta limitée tout au long de la période considérée. Les listes d'auditeurs publiées dans les annuaires de la $\mathrm{VI}^{\mathrm{e}}$ Section jusqu'en 1964/65 comprennent de 10 à 20 personnes. Même les rares chiffres donnés dans les rapports d'activités des annuaires des années 1970 ne montrent pas une augmentation sensible. La position marginale de la $\mathrm{VI}^{\mathrm{e}}$ Section dans l'enseignement supérieur peut 
expliquer cette stabilité relative de l'audience. Mais dans ce cercle restreint, le grand nombre d'étrangers venus surtout d'Amérique Latine, de Pologne et des pays européens voisins, était un signe du rayonnement international des activités du CRH ${ }^{5}$.

L'enseignement de l'École était toujours orienté vers la formation des chercheurs à la pratique de la recherche. E. Labrousse a exprimé les ambitions programmatiques de la $\mathrm{VI}^{\mathrm{e}}$ Section en la définissant comme une "véritable École normale de la Recherche, d'une recherche interdisciplinaire $»^{6}$. Dans le cas de l'histoire, cette ambition était limitée par la position à la fois marginale et ambiguë de l'École dans le système d'enseignement supérieur. Libre des obligations thématiques imposées par les concours du CAPES et de l'agrégation, l'enseignement et le diplôme de la $\mathrm{VI}^{\mathrm{e}}$ Section n'étaient tout simplement pas intégrés dans le système. Ses tentatives pour faire reconnaître son propre diplôme par les universités lors de la création du doctorat de recherche et du troisième cycle ont buté contre la méfiance des universités, jalouses de leur monopole du doctorat. Il en résulta une situation ambiguë, en ce qui concerne à la fois le niveau exigé pour les thèses préparées à la VIe Section et la valeur de ces diplômes sur le marché universitaire. Dans le cas de l'histoire, le poids des diplômes classiques - agrégation et doctorat d'État - restait prépondérant et le nombre de thèses de troisième cycle préparées à la division d'histoire reste faible : les 90 thèses répertoriées pour la période 1960-1974/75 ne représentent que $8,6 \%$ des thèses préparées à la $\mathrm{VI}^{\mathrm{e}}$ Section $^{7}$.

21 Contrairement aux ambitions affichées, les activités de recherche du CRH n'ont pas laissé de traces visibles dans la formation de troisième cycle des historiens. D'abord, on constate que les directeurs d'études engagés dans les enquêtes du Centre n'ont dirigé qu'un faible nombre de thèses (une vingtaine sur quatre vingt dix, soit $22 \%)^{8}$. Un indicateur encore plus sûr de l'intégration des travaux de formation à la recherche dans les enquêtes du CRH, nous est fourni par le nombre de chercheurs du CRH inscrits en troisième cycle. Pour la période 1960-1975, nous n'avons retrouvé que 15 chefs de travaux attachés au CRH (un bon tiers de ceux qui n'avaient pas soutenu une thèse à l'étranger ou qui n'étaient pas agrégés) ayant soutenu une thèse de troisième cycle ${ }^{9}$.

Par conséquent, le rapport thématique entre les recherches au $\mathrm{CRH}$ et les sujets de thèses n'est pas étroit. Bien qu'on retrouve les grandes orientations de la recherche et de l'enseignement historique à la $\mathrm{VI}^{\mathrm{e}}$ Section dans les sujets de thèses (surtout, la prépondérance des dix septième et dix huitième siècles français et des monographies locales ou régionales en histoire économique et sociale), le poids de choix marginaux reste fort: l'histoire politique, surtout de politique économique, des sujets classiques d'histoire des idées, n'ont cessé d'intéresser les étudiants de troisième cycle. On peut y déceler à la fois la trace de l'intérêt des étudiants étrangers pour l'histoire politique de leurs pays natals, surtout quand il s'agissait de pays du tiers monde, et l'effet de la politisation qui, à partir de 1968, se traduit par l'augmentation des sujets théoriques d'inspiration marxiste. 


\section{NOTES}

1. Brigitte Mazon, op. cit., p. 108-110 ; G. Gemelli, «La VI sezione dell'EPHE e l'unificazione delle scienze economico-sociali in Francia ", Inchiesta (1984), p. 138 et sv.

2. A cause de cette position marginale et passagère au CRH je n'ai pas mentionné dans les pages qui suivent ce groupe et son travail. Par cette exclusion, j'ai d'ailleurs évité certaines confusions qui résultent par exemple des divisions des sections du CNRS qui regroupe l'histoire ancienne dans la Section des civilisations classiques - reproduisant ainsi une liaison traditionnelle entre la philologie classique et l'histoire ancienne en France.

3. J'ai éliminé de cette comparaison les 8 chefs de travaux d'origine étrangère qui, de par leur âge au moment de leur recrutement, la nature de leurs diplômes et les règlements de l'administration française, ont suivi une carrière généralement plus lente que leurs collègues français.

4. Avec l'élargissement du personnel de la $\mathrm{VI}^{\mathrm{e}}$ Section, cette pratique a pesé de plus en plus sur le budget et sur la capacité d'innovation et d'ouverture de l'école. Malgré les réticences de la direction et une discussion sur ses effets négatifs, elle s'est poursuivie tout au long des années 1970.

5. Les étrangers étaient largement majoritaires à la VIe Section, ils représentaient $52 \%$ des étudiants inscrits à l'École en 1970 (VI Section de l'EPHE, budget 1971, archives EHESS, AJ 66, B 23).

6. Procès verbal de la réunion des directeurs d'études de la VI ${ }^{\mathrm{e}}$ Section, 24 novembre 1968. Procès-verbaux des assemblées de la VI ${ }^{\mathrm{e}}$ Section, t. 3, p. 94. Archives EHESS.

7. Les calculs ont été faits à partir des listes de thèses soutenues publiées dans les annuaires de la VIe Section et du fichier des thèses préparées à l'École. Je n'ai pris en considération que les thèses dirigées par un directeur d'études de la division d'histoire - en excluant tous les travaux de caractère historiographique préparés dans le domaine des aires culturelles.

8. Ce sont les historiens liés par leur enseignement à la $\mathrm{VI}^{\mathrm{e}}$ Section et leur travail individuel à «l'école des Annales » qui ont attiré la plupart des étudiants de troisième cycle. En tête, Vilar qui a dirigé 12 thèses soutenues entre 1960 et 1975, R. Romano qui en dirigea 11 entre 1966 et 1975 et Braudel avec 5 thèses après 1972 .

9. On peut ajouter trois autres thèses soutenues entre 1981 et 1985 par des chefs de travaux recrutés entre 1960 et 1975. 